\title{
Protolith age of Santa Maria Chico granulites dated on zircons from an associated amphibolite-facies granodiorite in southernmost Brazil
}

\author{
LÉO A. HARTMANN ${ }^{1}$, DUNYI LIU ${ }^{2}$, YENBIN WANG $^{2}$, HANS-JOACHIM MASSONNE ${ }^{3}$ \\ and JOÃO O.S. SANTOS ${ }^{4}$ \\ ${ }^{1}$ Instituto de Geociências, Universidade Federal do Rio Grande do Sul, Av. Bento Gonçalves, 9500 \\ 91501-970 Porto Alegre, Rio Grande do Sul, Brasil \\ ${ }^{2}$ Chinese Academy of Geological Sciences, 26 Baiwanzhuang Road, Beijing 100037, China \\ ${ }^{3}$ Institut für Mineralogie und Kristallchemie, Universität Stuttgart, Azenbergstr. 18, D-70174 Stuttgart, Germany \\ ${ }^{4}$ Centre for Global Targeting, University of Western Australia, Crawley, Perth 6009, WA, Australia \\ Manuscript received on January 17, 2007; accepted for publication on February 1, 2008; \\ contributed by LÉO A. HARTMANN*
}

\begin{abstract}
$\mathrm{U}-\mathrm{Pb}$ dating of zircon was undertaken with the Beijing SHRIMP II (sensitive high resolution ion microprobe) on an amphibolite facies granodiorite and an almandine-albite granulite from the Santa Maria Chico Granulitic Complex, southern Brazilian Shield. This work was also done to unravel protolith ages which are often hidden in the array of partly reset data. The obtained metamorphic ages of the granodiorite gneiss and the granulite are $2035 \pm 9 \mathrm{Ma}$ and $2006 \pm 3 \mathrm{Ma}$, respectively. These data are within the range of metamorphic ages determined in previous studies $(2022 \pm 18 \mathrm{Ma}$ and $2031 \pm 40 \mathrm{Ma})$. However, protolith ages for the granodiorite $(2366 \pm 8 \mathrm{Ma})$ and the granulite $(2489 \pm 6 \mathrm{Ma})$ were obtained which are outside the previously recognized range ( $>2510-2555 \mathrm{Ma})$. The magmatic protolith age of the granodiorite refers to a previously little known magmatic event in the shield. Further investigations may demonstrate that amphibolite facies zircon crystals are useful as a window into geological events in associated granulites, because zircon ages are blurred in the studied granulites.
\end{abstract}

Key words: protolith age, Paleoproterozoic, zircon geochronology, Santa Maria Chico Granulitic Complex, SHRIMP.

\section{INTRODUCTION}

The determination of protolith ages in granulite facies rocks is a major challenge in geochronology, because zircon - the most important mineral in geochronology - undergoes extensive recrystallization and partial resetting of ages during high temperature metamorphism, commonly $\geq 800^{\circ} \mathrm{C}$ at $25-40 \mathrm{~km}$ depth for a long period of time ( $\geq$ one million years). The use of a sensitive high-resolution ion microprobe (SHRIMP) for analyses of spots with $30 \mu \mathrm{m}$ diameter in zircon crystals from gran-

\footnotetext{
*Member Academia Brasileira de Ciências

Correspondence to: Léo A. Hartmann

E-mail: leo.hartmann@ufrgs.br
}

ulites has been a major advance in the understanding of zircon ages from high grade terrains (Friend and Kinny 1995, Hartmann et al. 1999, Santos et al. 2003, Silva 2006). However, a homogeneous, relict magmatic portion in zircon that can be selected for dating is rarely available. Although the resilient nature of zircon is apparent in crystals which experienced repeated granulite facies metamorphism, the interpretation of ages obtained by ion microprobe is often difficult because of the blurring of U-Pb isotopic compositions (Hartmann et al. 2000b), particularly for zircons with high $\mathrm{U}$ concentration.

In the present paper, the dating of zircon in structurally concordant amphibolite facies rocks is a step to- 
wards the solution of this problem whenever such rocks occur in the same complex. The rationale is that rims and unaltered cores are commonly present in crystals submitted to metamorphic conditions (e.g., $600-700^{\circ} \mathrm{C}$, 3-5 $\mathrm{Kb}$ ) less severe than those of the granulite facies. Thus, one amphibolite facies and one granulite facies rock from the Paleoproterozoic Santa Maria Chico Granulitic Complex, Brazil, were dated with the SHRIMP II in Beijing. These new results are integrated with previous data on zircon crystals (Table I) from the same complex (Hartmann et al. 1999) obtained at the SHRIMP II in Perth. This geochronological method is capable of establishing the protolith age recorded in the cores of zircon crystals. Therefore, the method can help to elucidate the geological history of complex granulite-amphibolite facies terrains whenever the rocks underwent the same structural evolution as in the present case. Other geological relationships require careful evaluation before interpretation of coeval evolution of the rocks.

\section{GEOLOGICAL RELATIONSHIPS}

The Santa Maria Chico Granulitic Complex in southern Brazil (Fig. 1) was studied because it attained deep crustal conditions of metamorphic equilibration $\left(800^{\circ} \mathrm{C}\right.$ and $10 \mathrm{Kbar}$, Hartmann 1998; early stage $9 \mathrm{kbar}$ and $800^{\circ} \mathrm{C}$, late stage $7 \mathrm{kbar}$ and $700^{\circ} \mathrm{C}$, Massonne et al. 2001. Zircon isotopic compositions are extensively reset. All other granulite complexes which have been investigated in South America are located farther north in the Brazilian Shield. In addition, large sections of amphibolite facies rocks associated with smaller areas of granulites are exposed and most of them experienced lower pressure conditions (5-7 Kbar). The intense recrystallization of the Santa Maria Chico granulites has led to widespread, equilibrium granulite facies assemblages (Hartmann 1998, Hartmann et al. 1999). Rocks in the complex are bimodal. Trondhjemites dominate over mafic garnet granulites, pyroxenite, spinel lherzolite and sillimanite gneiss. The dominant structure is a subvertical foliation striking E-W in the southern part and NW in the northern part of the complex.

Field mapping in 2001-2002 led to the recognition of a granodiorite gneiss $\left(>25 \mathrm{~km}^{2}\right)$, which shows the same deformation as the enclosing granulite complex, the S1 high-grade subvertical foliation. The hypothesis that the rocks, showing metamorphic assemblages of the amphibolite and granulite facies, were deformed during the same event can be tested by dating zircon from the amphibolite-facies gneiss associated with the granulite complex, although the blurring of ages of granulite zircons may diminish the significance of the test. The minerals of the gneiss are in granoblastic equilibrium and no remnant magmatic texture or mineral is discernible under the petrographic microscope, a similar relationship observed on granulite facies rocks of the complex.

\section{GEOCHRONOLOGY}

In fact, the main objective was to determine the magmatic age of the protoliths of the rocks, but a necessary condition to test the proposed method was that the amphibolite facies and granulite facies metamorphic events are synchronous. Otherwise, additional complexity in the geological relationships would hamper the interpretations. Petrographic examination of the zircon crystals from the granodiorite gneiss (sample 1) reveals welldeveloped cores and rims in 100-200 $\mu \mathrm{m}$ sized crystals with rounded terminations and aspect ratios of $3: 1$. Thus, separate ages could be determined by $30-\mu \mathrm{m}$ spot SHRIMP dating. Zircon crystals in the almandine-albite granulite (sample 2) are smaller $(5-100 \mu \mathrm{m})$, rounded, and show more complex internal structure.

Zircon crystals $(\mathrm{n}=80)$ were separated from $2 \mathrm{~kg}$ of each rock (samples 1 and 2) by mechanical and magnetic processes, mounted on an epoxy disc, polished and covered with gold for isotopic determinations guided by optical photographs. The Beijing SHRIMP II analytical techniques employed are similar to those reported by Smith et al. (1998).

The indiscriminate use of $\mathrm{Th} / \mathrm{U}$ ratios of zircon as petrogenetic indicators can lead to gross misinterpretation of the magmatic versus metamorphic environment of zircon crystallization (Möller et al. 2003). In the present study, however, the geological and petrographic characteristics of the rocks and the consistency of results from previous studies (Hartmann et al. 1999) and from the present investigation lead us to consider the $\mathrm{Th} / \mathrm{U}$ ratios of the analyzed zircon as reliable indicators of magmatic versus metamorphic crystallization.

The analyses of 41 spots in 38 zircon crystals from sample 1 (field number BRA44), granodiorite gneiss, re- 
TABLE I

Geochronological data available on Santa Maria Chico Granulitic Complex.

\begin{tabular}{|c|c|c|c|c|c|}
\hline Rock & Material & Method & Observation & Age, Ma & Reference \\
\hline Mafic granulite & Zircon & SHRIMP & $\begin{array}{l}\text { Core } \\
\text { Rim }\end{array}$ & $\begin{array}{c}2509 \\
2022 \pm 18\end{array}$ & Hartmann et al. (1999) \\
\hline Trondhjemite & Zircon & SHRIMP & $\begin{array}{l}\text { Core } \\
\text { Rim }\end{array}$ & $\begin{array}{c}2553 \\
2031 \pm 40\end{array}$ & Hartmann et al. (1999) \\
\hline $\begin{array}{l}\text { Granodiorite } \\
\text { gneiss }\end{array}$ & Zircon & SHRIMP & $\begin{array}{l}\text { Core } \\
\text { Rim }\end{array}$ & $\begin{array}{l}2366 \pm 8 \\
2035 \pm 9\end{array}$ & This work \\
\hline $\begin{array}{l}\text { Almandine- } \\
\text { albite granulite }\end{array}$ & Zircon & SHRIMP & $\begin{array}{l}\text { Core } \\
\text { Rim }\end{array}$ & $\begin{array}{l}2489 \pm 6 \\
2006 \pm 3\end{array}$ & This work \\
\hline $\begin{array}{l}\text { Sillimanite } \\
\text { gneiss }\end{array}$ & $\begin{array}{l}\text { Garnet, } \\
\text { plagioclase, } \\
\text { total rock }\end{array}$ & $\begin{array}{l}\text { Sm-Nd } \\
\text { isochron }\end{array}$ & & ca. 2100 & Hartmann (1998) \\
\hline $\begin{array}{l}\text { Mafic } \\
\text { granulite }\end{array}$ & Whole rock & $\begin{array}{l}\text { Model Nd } \\
\mathrm{T}_{\mathrm{DM}}\end{array}$ & & ca. 2600 & Hartmann (1998) \\
\hline Trondhjemite & Whole rock & $\begin{array}{l}\text { Model Nd } \\
\mathrm{T}_{\mathrm{DM}}\end{array}$ & & ca. 2300 & Hartmann (1998) \\
\hline $\begin{array}{l}\text { Mafic and } \\
\text { felsic rocks }\end{array}$ & Whole rock & $\begin{array}{l}\mathrm{Pb}-\mathrm{Pb} \\
\text { isochron }\end{array}$ & & $2550 \pm 150$ & Soliani Jr. (1986) \\
\hline $\begin{array}{l}\text { Intrusive } \\
583 \pm 2 \mathrm{Ma} \\
\text { S. Antônio } \\
\text { monzogranite }\end{array}$ & $\begin{array}{l}\text { Zircon } \\
\text { (xenocryst) }\end{array}$ & $\begin{array}{l}\mathrm{Pb}-\mathrm{Pb} \\
\text { evaporation }\end{array}$ & $\begin{array}{l}\text { S. Antonio } \\
\text { Granite age } \\
583 \pm 2 \mathrm{Ma}\end{array}$ & ca. 2350 & Gastal and Lafon (2001) \\
\hline
\end{tabular}

sulted in ${ }^{207} \mathrm{~Pb} /{ }^{206} \mathrm{~Pb}$ age spread between $2689 \mathrm{Ma}$ and $2002 \mathrm{Ma}$ (Table II, Fig. 3) with two well-defined age groups, at $2366 \pm 8 \mathrm{Ma}$ and $2035 \pm 9 \mathrm{Ma}$ and possibly a third group at $\sim 2200 \mathrm{Ma}$. Th/U ratios (Fig. 3) are near 0.4 for the 2366 Ma group, and these are commonly accepted as magmatic compositions (Vavra et al. 1999, Hartmann et al. 2000a) and 0.01 for the $2035 \mathrm{Ma}$ age group, which are usually considered as metamorphic ratios. The nature of the $\sim 2200 \mathrm{Ma}$ age requires further investigations. The magmatic age of the granodiorite gneiss is interpreted as $2366 \pm 8 \mathrm{Ma}$ and the age of amphibolite facies recrystallization as $2035 \pm 9 \mathrm{Ma}$. $\mathrm{Pb}$ $\mathrm{Pb}$ evaporation ages near $2.35 \mathrm{Ma}$ (minimum age, Gastal and Lafon 2001) are known from zircon xenocrysts in the Santo Antonio Granite, emplaced in the granulite complex at $583 \pm 2 \mathrm{Ma}$ and located $10 \mathrm{~km}$ from the studied granodiorite gneiss sample. The presently dated granodiorite is one of the possible sources of the $2.35 \mathrm{Ga}$ xenocrysts present in the Santo Antonio Granite. A total of 60 analyses were made on zircon from sample 1, but 19 were discarded because the crystals have extremely low
$\mathrm{U}(6-10 \mathrm{ppm})$ and required extraordinary high common $\mathrm{Pb}$ corrections (1.19-9.46\%).

In sample 2 (field number SMC1), almandine-albite granulite, the analyses of 17 spots in 15 zircon crystals yielded a ${ }^{207} \mathrm{~Pb} /{ }^{206} \mathrm{~Pb}$ age spread between 2578 and 1998 Ma (Table II, Fig. 3). The youngest age cluster (2006 \pm 3 $\mathrm{Ma}$ ) is interpreted as the age of granulite facies metamorphism, because $\mathrm{Th} / \mathrm{U}$ ratios are low (0.05) and the spot analyses were determined on rims of zircon crystals. As sample 2 is probably a metagraywacke, because of its mineralogy (mostly almandine + albite + quartz), the oldest age cluster ( $2489 \pm 6 \mathrm{Ma}$ ) can be interpreted as referring to the age of the source of detrital zircon crystals. However, the ages between $2489 \mathrm{Ma}$ and $2006 \mathrm{Ma}$ may correspond either to different sources of the detrital zircon or to deformational episodes of the metasediment. These geological events cannot be defined more precisely in time with the present data set, but it is clear that the sedimentary basin is older than $2006 \mathrm{Ma}$, because this is the age of granulite facies metamorphism of the Santa Maria Chico granulites as previously determined by 
TABLE II

U-Pb zircon SHRIMP II isotopic data from Santa Maria Chico Complex.

\begin{tabular}{|c|c|c|c|c|c|c|c|c|c|c|c|c|}
\hline \multirow[b]{2}{*}{ Spot } & \multirow[b]{2}{*}{$\begin{array}{c}\mathrm{U} \\
\mathrm{ppm}\end{array}$} & \multirow[b]{2}{*}{$\begin{array}{c}\text { Th } \\
\text { ppm }\end{array}$} & \multirow[b]{2}{*}{$\begin{array}{l}\text { Th } \\
\text { U }\end{array}$} & \multirow[b]{2}{*}{$\begin{array}{c}4 \mathrm{f} 206 \\
(\%)\end{array}$} & \multicolumn{5}{|c|}{ Isotopic ratios } & \multicolumn{2}{|c|}{ Ages } & \multirow[b]{2}{*}{$\begin{array}{c}\text { Disc. } \\
\%\end{array}$} \\
\hline & & & & & ${ }^{207} \mathrm{~Pb} /{ }^{206} \mathrm{~Pb}$ & ${ }^{208} \mathrm{~Pb} /{ }^{206} \mathrm{~Pb}$ & ${ }^{206} \mathrm{~Pb} /{ }^{238} \mathrm{U}$ & ${ }^{207} \mathrm{~Pb} /{ }^{235} \mathrm{U}$ & ${ }^{208} \mathrm{~Pb} /{ }^{232} \mathrm{Th}$ & ${ }^{207} \mathrm{~Pb} /{ }^{206} \mathrm{~Pb}$ & ${ }^{206} \mathrm{~Pb} /{ }^{238} \mathrm{U}$ & \\
\hline \multicolumn{13}{|c|}{ Sample 1 (BRA44), granodiorite gneiss } \\
\hline $6.1 \mathrm{~A}$ & 34 & 16 & 0.48 & 0.06 & $0.15133 \pm 1.17$ & $0.1429 \pm 1.55$ & $0.4238 \pm 0.93$ & $8.8418 \pm 1.49$ & $0.1258 \pm 1.92$ & $2361 \pm 20$ & $2278 \pm 18$ & 4 \\
\hline $20.1 \mathrm{~A}$ & 13 & 1 & 0.08 & 0.81 & $0.11978 \pm 3.02$ & $0.0314 \pm 5.88$ & $0.3385 \pm 1.55$ & $5.5911 \pm 3.40$ & & $1953 \pm 54$ & $1880 \pm 25$ & 4 \\
\hline $21.1 \mathrm{~A}$ & 7 & 1 & 0.09 & 0.79 & $0.12233 \pm 4.22$ & $0.0324 \pm 7.37$ & $0.3610 \pm 2.09$ & $6.0895 \pm 4.72$ & & $1990 \pm 75$ & $1987 \pm 36$ & 0 \\
\hline $23.1 \mathrm{~A}$ & 6 & 1 & 0.10 & 0.00 & $0.12829 \pm 3.85$ & $0.0403 \pm 8.22$ & $0.3608 \pm 2.18$ & $6.3826 \pm 4.43$ & & $2075 \pm 68$ & $1986 \pm 37$ & 4 \\
\hline $24.1 \mathrm{~A}$ & 7 & 1 & 0.08 & 0.86 & $0.13008 \pm 4.22$ & $0.0438 \pm 7.06$ & $0.3504 \pm 2.24$ & $6.2841 \pm 4.77$ & & $2099 \pm 74$ & $1936 \pm 37$ & 8 \\
\hline 22.1 & 63 & 42 & 0.68 & 0.38 & $0.12254 \pm 1.32$ & $0.1966 \pm 1.49$ & $0.3728 \pm 0.72$ & $6.2989 \pm 1.50$ & $0.1032 \pm 2.23$ & $1994 \pm 24$ & $2043 \pm 13$ & -2 \\
\hline 27.1 & 81 & 33 & 0.42 & 0.11 & $0.15157 \pm 1.05$ & $0.1177 \pm 1.08$ & $0.4446 \pm 0.61$ & $9.2914 \pm 1.21$ & $0.1225 \pm 1.46$ & $2364 \pm 18$ & $2371 \pm 12$ & 0 \\
\hline 28.1 & 119 & 45 & 0.39 & 0.12 & $0.14711 \pm 1.26$ & $0.1109 \pm 1.05$ & $0.4079 \pm 0.52$ & $8.2729 \pm 1.36$ & $0.1133 \pm 1.47$ & $2313 \pm 22$ & $2205 \pm 10$ & 5 \\
\hline 29.1 & 82 & 31 & 0.39 & 0.07 & $0.15125 \pm 0.77$ & $0.1119 \pm 1.12$ & $0.4430 \pm 1.33$ & $9.2380 \pm 1.53$ & $0.1253 \pm 1.89$ & $2360 \pm 13$ & $2364 \pm 26$ & 0 \\
\hline 30.1 & 8 & 1 & 0.11 & 0.00 & $0.13148 \pm 2.94$ & $0.0477 \pm 5.97$ & $0.3681 \pm 1.99$ & $6.6727 \pm 3.55$ & $0.1780 \pm 9.58$ & $2118 \pm 52$ & $2020 \pm 34$ & 5 \\
\hline 32.1 & 20 & 11 & 0.56 & 0.95 & $0.11959 \pm 2.09$ & $0.1661 \pm 2.08$ & $0.3611 \pm 1.24$ & $5.9538 \pm 2.43$ & $0.0941 \pm 3.27$ & $1950 \pm 37$ & $1987 \pm 21$ & -2 \\
\hline 34.1 & 7 & 1 & 0.08 & 0.00 & $0.13881 \pm 3.68$ & $0.0387 \pm 8.45$ & $0.4122 \pm 2.55$ & $7.8884 \pm 4.47$ & $0.3064 \pm 13.50$ & $2212 \pm 64$ & $2225 \pm 48$ & -1 \\
\hline 35.1 & 13 & 1 & 0.07 & 0.34 & $0.12381 \pm 2.49$ & $0.0317 \pm 5.63$ & $0.3845 \pm 1.74$ & $6.5639 \pm 3.04$ & & $2012 \pm 44$ & $2097 \pm 31$ & -4 \\
\hline 36.1 & 61 & 53 & 0.89 & 0.20 & $0.18428 \pm 0.86$ & $0.2460 \pm 0.81$ & $0.5527 \pm 0.68$ & $14.0436 \pm 1.10$ & $0.1505 \pm 1.21$ & $2692 \pm 14$ & $2836 \pm 16$ & -5 \\
\hline 36.2 & 58 & 55 & 0.98 & 0.29 & $0.14770 \pm 1.07$ & $0.2786 \pm 0.95$ & $0.4581 \pm 0.86$ & $9.3287 \pm 1.37$ & $0.1269 \pm 1.49$ & $2319 \pm 18$ & $2431 \pm 17$ & -5 \\
\hline 37.1 & 15 & 1 & 0.07 & 0.00 & $0.12797 \pm 2.36$ & $0.0316 \pm 5.30$ & $0.3768 \pm 1.49$ & $6.6489 \pm 2.79$ & $0.2052 \pm 11.74$ & $2070 \pm 42$ & $2061 \pm 26$ & 0 \\
\hline 38.1 & 7 & 1 & 0.09 & 0.65 & $0.13057 \pm 3.04$ & $0.0365 \pm 7.06$ & $0.3671 \pm 2.08$ & $6.6082 \pm 3.68$ & $0.0925 \pm 9.59$ & $2106 \pm 53$ & $2016 \pm 36$ & 4 \\
\hline 39.1 & 7 & 1 & 0.10 & 0.14 & $0.12844 \pm 2.88$ & $0.0394 \pm 6.63$ & $0.3834 \pm 2.02$ & $6.7896 \pm 3.52$ & & $2077 \pm 51$ & $2092 \pm 36$ & -1 \\
\hline 40.1 & 55 & 25 & 0.48 & 0.20 & $0.15312 \pm 0.97$ & $0.1363 \pm 1.25$ & $0.4615 \pm 0.75$ & $9.7425 \pm 1.22$ & $0.1278 \pm 1.82$ & $2381 \pm 16$ & $2446 \pm 15$ & -3 \\
\hline 43.1 & 7 & 1 & 0.12 & 0.98 & $0.11751 \pm 5.01$ & $0.0477 \pm 6.10$ & $0.3699 \pm 2.11$ & $5.9923 \pm 5.43$ & & $1919 \pm 90$ & $2029 \pm 37$ & -6 \\
\hline 43.2 & 80 & 21 & 0.27 & 0.09 & $0.15234 \pm 1.16$ & $0.0769 \pm 2.30$ & $0.4520 \pm 1.25$ & $9.4946 \pm 1.71$ & $0.1273 \pm 2.94$ & $2372 \pm 20$ & $2404 \pm 25$ & -1 \\
\hline 45.1 & 11 & 1 & 0.07 & 0.00 & $0.12275 \pm 2.35$ & $0.0300 \pm 6.17$ & $0.3724 \pm 1.66$ & $6.3024 \pm 2.88$ & & $1997 \pm 42$ & $2041 \pm 29$ & -2 \\
\hline 46.1 & 32 & 15 & 0.48 & 0.33 & $0.13272 \pm 1.80$ & $0.1401 \pm 1.73$ & $0.3792 \pm 0.98$ & $6.9389 \pm 2.06$ & $0.1041 \pm 2.93$ & $2134 \pm 32$ & $2072 \pm 17$ & 3 \\
\hline 47.1 & 9 & 1 & 0.15 & 0.60 & $0.12646 \pm 3.35$ & $0.0540 \pm 5.43$ & $0.3503 \pm 1.87$ & $6.1071 \pm 3.84$ & & $2049 \pm 59$ & $1936 \pm 31$ & 6 \\
\hline 48.1 & 7 & 1 & 0.09 & 0.27 & $0.12655 \pm 3.24$ & $0.0403 \pm 7.08$ & $0.3765 \pm 3.05$ & $6.5699 \pm 4.45$ & & $2051 \pm 57$ & $2060 \pm 54$ & 0 \\
\hline 49.1 & 112 & 59 & 0.54 & 0.14 & $0.15214 \pm 0.99$ & $0.1514 \pm 0.86$ & $0.4227 \pm 0.53$ & $8.8659 \pm 1.12$ & $0.1164 \pm 1.20$ & $2370 \pm 17$ & $2273 \pm 10$ & 4 \\
\hline 50.1 & 8 & 1 & 0.08 & 0.00 & $0.13034 \pm 4.16$ & $0.0335 \pm 7.74$ & $0.3569 \pm 2.42$ & $6.4132 \pm 4.81$ & & $2103 \pm 73$ & $1967 \pm 41$ & 6 \\
\hline 51.1 & 64 & 49 & 0.79 & 0.00 & $0.15296 \pm 0.82$ & $0.2214 \pm 0.93$ & $0.4596 \pm 0.69$ & $9.6941 \pm 1.07$ & $0.1293 \pm 1.16$ & $2379 \pm 14$ & $2438 \pm 14$ & -2 \\
\hline 52.1 & 164 & 64 & 0.40 & 0.16 & $0.13778 \pm 0.83$ & $0.1210 \pm 1.15$ & $0.4113 \pm 0.49$ & $7.8141 \pm 0.96$ & $0.1205 \pm 1.51$ & $2199 \pm 14$ & $2221 \pm 9$ & -1 \\
\hline 54.1 & 130 & 39 & 0.31 & 0.07 & $0.15331 \pm 0.71$ & $0.0853 \pm 1.20$ & $0.4174 \pm 0.59$ & $8.8226 \pm 0.92$ & $0.1119 \pm 1.50$ & $2383 \pm 12$ & $2249 \pm 11$ & 6 \\
\hline 55.1 & 81 & 31 & 0.39 & 0.09 & $0.14319 \pm 1.22$ & $0.1108 \pm 1.13$ & $0.4242 \pm 0.62$ & $8.3756 \pm 1.37$ & $0.1181 \pm 1.44$ & $2266 \pm 21$ & $2280 \pm 12$ & -1 \\
\hline 56.1 & 116 & 49 & 0.44 & 0.18 & $0.15716 \pm 0.68$ & $0.1253 \pm 0.90$ & $0.4476 \pm 0.53$ & $9.6985 \pm 0.87$ & $0.1244 \pm 1.45$ & $2425 \pm 12$ & $2384 \pm 11$ & 2 \\
\hline 57.1 & 92 & 38 & 0.43 & 0.04 & $0.14428 \pm 1.58$ & $0.1231 \pm 1.04$ & $0.4165 \pm 1.01$ & $8.2858 \pm 1.88$ & $0.1187 \pm 1.48$ & $2279 \pm 27$ & $2245 \pm 19$ & 2 \\
\hline 59.1 & 74 & 36 & 0.50 & 0.01 & $0.15823 \pm 0.84$ & $0.1439 \pm 1.72$ & $0.3930 \pm 0.74$ & $8.5751 \pm 1.11$ & $0.1120 \pm 1.88$ & $2437 \pm 14$ & $2137 \pm 13$ & 12 \\
\hline 60.1 & 195 & 87 & 0.46 & 0.04 & $0.14298 \pm 1.16$ & $0.1345 \pm 0.72$ & $0.4037 \pm 0.56$ & $7.9593 \pm 1.29$ & $0.1169 \pm 0.95$ & $2264 \pm 20$ & $2186 \pm 10$ & 3 \\
\hline 61.1 & 416 & 108 & 0.27 & 0.03 & $0.15075 \pm 0.44$ & $0.0767 \pm 0.61$ & $0.4282 \pm 0.29$ & $8.8995 \pm 0.52$ & $0.1208 \pm 0.74$ & $2354 \pm 7$ & $2297 \pm 6$ & 2 \\
\hline 61.2 & 54 & 8 & 0.15 & 0.00 & $0.16607 \pm 0.85$ & $0.0425 \pm 2.09$ & $0.5027 \pm 0.78$ & $11.5107 \pm 1.15$ & $0.1414 \pm 2.57$ & $2518 \pm 14$ & $2625 \pm 17$ & -4 \\
\hline 62.1 & 315 & 132 & 0.43 & 0.11 & $0.14664 \pm 1.13$ & $0.1267 \pm 0.93$ & $0.4048 \pm 0.31$ & $8.1850 \pm 1.17$ & $0.1163 \pm 1.09$ & $2307 \pm 19$ & $2191 \pm 6$ & 5 \\
\hline 63.1 & 68 & 48 & 0.73 & 0.00 & $0.13384 \pm 1.10$ & $0.2119 \pm 1.22$ & $0.3784 \pm 0.93$ & $6.9823 \pm 1.44$ & $0.1107 \pm 1.80$ & $2149 \pm 19$ & $2069 \pm 16$ & 4 \\
\hline 64.1 & 74 & 29 & 0.41 & 0.17 & $0.13248 \pm 1.13$ & $0.1238 \pm 1.37$ & $0.3498 \pm 0.79$ & $6.3891 \pm 1.38$ & $0.1029 \pm 1.65$ & $2131 \pm 20$ & $1934 \pm 13$ & 9 \\
\hline$x-6$ & 38 & 32 & 0.88 & 0.15 & $0.13008 \pm 2.02$ & $0.2593 \pm 1.92$ & $0.3741 \pm 1.60$ & $6.7103 \pm 2.58$ & $0.1085 \pm 2.62$ & $2099 \pm 35$ & $2049 \pm 28$ & 2 \\
\hline \multicolumn{13}{|c|}{ Sample 2 (SMC1), almandine-albite granulite } \\
\hline $6-1$ & 270 & 13 & 0.05 & 0.03 & $0.12451 \pm 0.80$ & $0.0148 \pm 1.73$ & $0.3647 \pm 1.38$ & $6.2604 \pm 1.60$ & $0.1116 \pm 2.34$ & $2022 \pm 14$ & $2004 \pm 24$ & 1 \\
\hline 6-2-1 & 220 & 158 & 0.74 & 0.08 & $0.16483 \pm 0.46$ & $0.2173 \pm 1.23$ & $0.4585 \pm 1.48$ & $10.4212 \pm 1.55$ & $0.1343 \pm 1.94$ & $2506 \pm 8$ & $2433 \pm 30$ & 3 \\
\hline $6-2-2$ & 161 & 116 & 0.75 & 0.00 & $0.15636 \pm 2.58$ & $0.2088 \pm 0.61$ & $0.4647 \pm 1.58$ & $10.0183 \pm 3.02$ & $0.1299 \pm 1.73$ & $2417 \pm 44$ & $2460 \pm 32$ & -2 \\
\hline 6-3 & 195 & 148 & 0.79 & 0.00 & $0.16010 \pm 0.86$ & $0.2299 \pm 1.07$ & $0.4707 \pm 1.43$ & $10.3907 \pm 1.67$ & $0.1377 \pm 2.04$ & $2457 \pm 15$ & $2487 \pm 29$ & -1 \\
\hline 6-4 & 226 & 28 & 0.13 & 0.06 & $0.14093 \pm 1.41$ & $0.0381 \pm 1.13$ & $0.4112 \pm 1.73$ & $7.9898 \pm 2.23$ & $0.1221 \pm 2.13$ & $2239 \pm 24$ & $2220 \pm 32$ & 1 \\
\hline $6-5$ & 225 & 42 & 0.19 & 0.00 & $0.12282 \pm 0.52$ & $0.0552 \pm 1.01$ & $0.3677 \pm 1.40$ & $6.2272 \pm 1.49$ & $0.1044 \pm 1.79$ & $1998 \pm 9$ & $2019 \pm 24$ & -1 \\
\hline 6-6 & 404 & 7 & 0.02 & 0.00 & $0.14692 \pm 0.96$ & $0.0052 \pm 3.34$ & $0.4411 \pm 1.63$ & $8.9350 \pm 1.89$ & $0.1254 \pm 4.17$ & $2310 \pm 16$ & $2355 \pm 32$ & -2 \\
\hline 6-7 & 290 & 120 & 0.43 & 0.07 & $0.15976 \pm 2.09$ & $0.1451 \pm 1.02$ & $0.4123 \pm 2.03$ & $9.0829 \pm 2.91$ & $0.1394 \pm 2.30$ & $2453 \pm 35$ & $2226 \pm 38$ & 9 \\
\hline 6-8 & 211 & 150 & 0.74 & 0.00 & $0.13757 \pm 0.94$ & $0.2030 \pm 1.28$ & $0.4078 \pm 2.23$ & $7.7351 \pm 2.42$ & $0.1125 \pm 2.59$ & $2197 \pm 16$ & $2205 \pm 42$ & 0 \\
\hline 6-9 & 614 & 286 & 0.48 & 0.04 & $0.15189 \pm 0.82$ & $0.1340 \pm 2.14$ & $0.4338 \pm 1.39$ & $9.0852 \pm 1.61$ & $0.1207 \pm 2.63$ & $2367 \pm 14$ & $2323 \pm 27$ & 2 \\
\hline $6-10$ & 61 & 31 & 0.53 & 0.26 & $0.16505 \pm 1.52$ & $0.1511 \pm 2.64$ & $0.4720 \pm 1.61$ & $10.7400 \pm 2.22$ & $0.1357 \pm 3.16$ & $2508 \pm 26$ & $2492 \pm 33$ & 1 \\
\hline $6-11$ & 182 & 34 & 0.19 & 0.34 & $0.17211 \pm 0.62$ & $0.0739 \pm 0.98$ & $0.4557 \pm 2.35$ & $10.8138 \pm 2.43$ & $0.1777 \pm 2.63$ & $2578 \pm 10$ & $2420 \pm 47$ & 6 \\
\hline 6-12-1 & 201 & 31 & 0.16 & 0.00 & $0.12359 \pm 0.68$ & $0.0469 \pm 1.29$ & $0.3633 \pm 1.42$ & $6.1901 \pm 1.58$ & $0.1071 \pm 2.03$ & $2009 \pm 12$ & $1998 \pm 24$ & 1 \\
\hline 6-12-2 & 479 & 171 & 0.37 & 0.01 & $0.13325 \pm 0.65$ & $0.1035 \pm 1.13$ & $0.3843 \pm 1.98$ & $7.0615 \pm 2.09$ & $0.1079 \pm 2.30$ & $2141 \pm 11$ & $2097 \pm 35$ & 2 \\
\hline $6-13$ & 222 & 25 & 0.12 & 0.79 & $0.13138 \pm 1.74$ & $0.0582 \pm 1.14$ & $0.3680 \pm 1.43$ & $6.6661 \pm 2.25$ & $0.1841 \pm 1.91$ & $2117 \pm 30$ & $2020 \pm 25$ & 5 \\
\hline $6-14$ & 158 & 97 & 0.63 & 0.06 & $0.15450 \pm 1.03$ & $0.1943 \pm 1.09$ & $0.4196 \pm 1.46$ & $8.9389 \pm 1.78$ & $0.1291 \pm 1.86$ & $2396 \pm 17$ & $2259 \pm 28$ & 6 \\
\hline $6-15$ & 345 & 195 & 0.58 & 0.00 & $0.15489 \pm 0.76$ & $0.1652 \pm 1.34$ & $0.4362 \pm 1.40$ & $9.3144 \pm 1.59$ & $0.1237 \pm 2.02$ & $2401 \pm 13$ & $2333 \pm 27$ & 3 \\
\hline
\end{tabular}

Notes: Isotopic ratios errors in \%. All $\mathrm{Pb}$ in ratios are radiogenic component, all corrected for ${ }^{204} \mathrm{~Pb}$. disc. $=$ discordance, as $100-100\left\{\mathrm{t}\left[{ }^{206} \mathrm{~Pb} /{ }^{238} \mathrm{U}\right] / \mathrm{t}\left[{ }^{207} \mathrm{~Pb} /{ }^{206} \mathrm{~Pb}\right]\right\}$. $4 \mathrm{f} 206=\left(\right.$ common $\left.{ }^{206} \mathrm{~Pb}\right) /\left(\right.$ total measured $\left.{ }^{206} \mathrm{~Pb}\right)$ based on measured ${ }^{204} \mathrm{~Pb}$ or ${ }^{208} \mathrm{~Pb}(8 \mathrm{f} 206)$. Uncertainties are $1 \sigma$. 


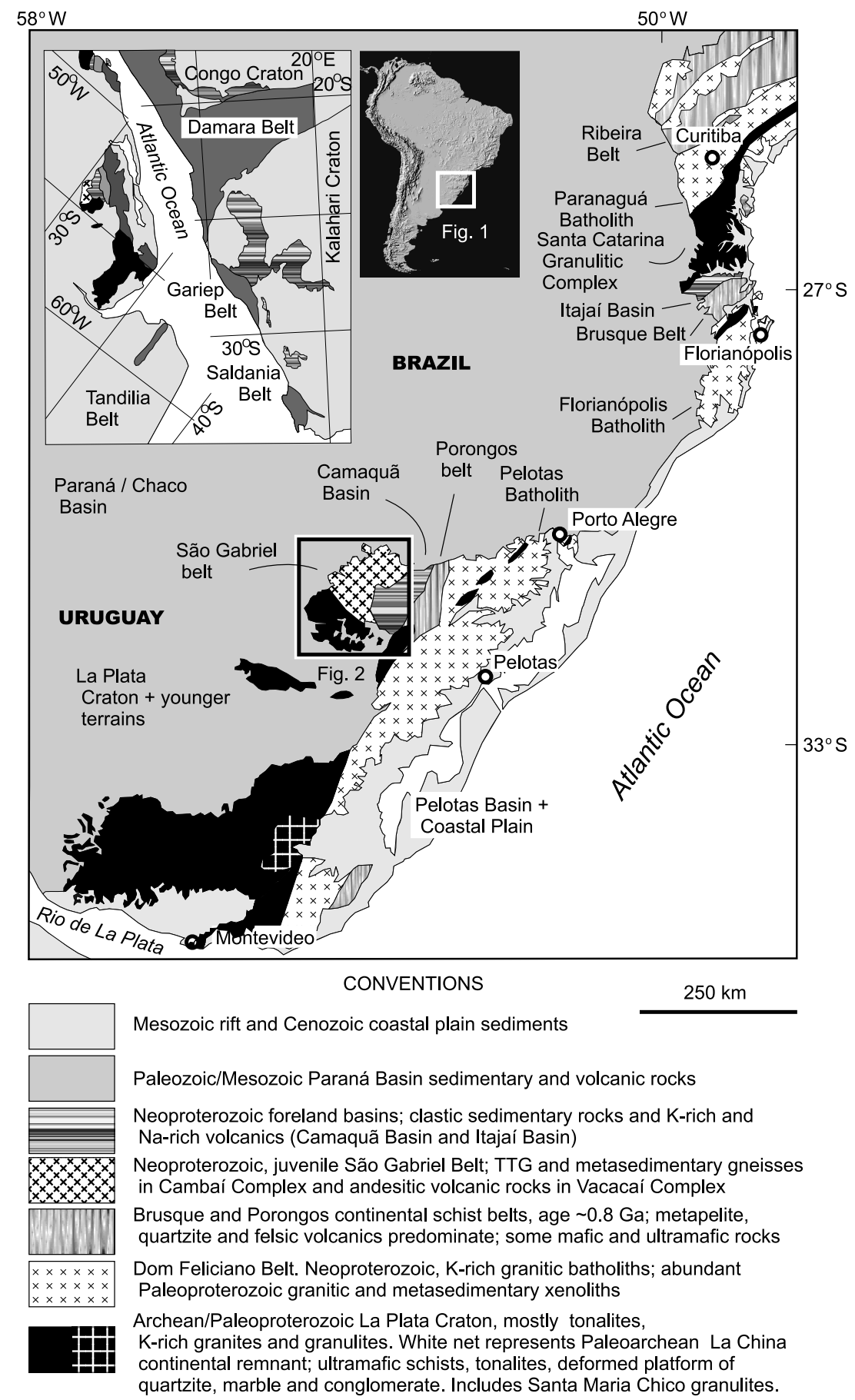

Fig. 1 - Position of studied granulitic complex in relation to regional geotectonic units.

Hartmann et al. (1999) at $2022 \pm 18 \mathrm{Ma}$ and $2031 \pm 40$ Ma for two ortho-granulites (Table I). The amphibolite facies event (sample 1) and granulite facies event (sample 2), as dated in this investigation, were therefore syn- chronous within error of the previously determined age of the granulite facies event, because both formed $M_{1}$ mineral assemblages at $2035 \pm 9 \mathrm{Ma}$ and $2006 \pm 3 \mathrm{Ma}$. Two Archean ages at $2688 \mathrm{Ma}$ and $2559 \mathrm{Ma}$ in sample 


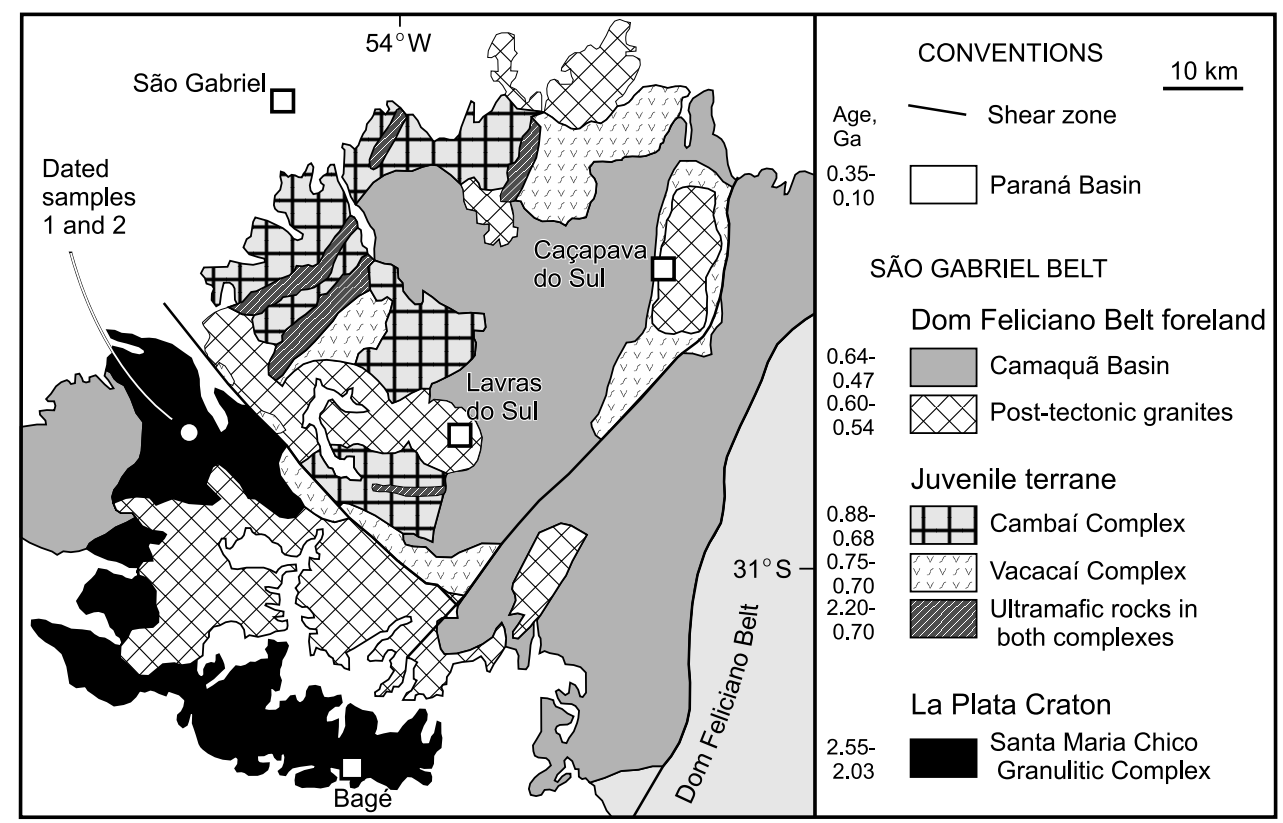

Fig. 2 - Geological map of the São Gabriel Belt in the southern Brazilian Shield. Location of studied samples 1 and 2 indicated.

1 are xenocrysts from a presumed, unknown basement. The granodiorite gneiss is therefore a window into processes older than $2.0 \mathrm{Ga}$.

\section{INTERPRETATIONS AND CONCLUSIONS}

A nearly continuous spread of zircon SHRIMP U-Pb ages was obtained by Hartmann et al. (1999) between $2.55 \mathrm{Ga}$ and $2.02 \mathrm{Ga}$ in two orthogranulites from the Santa Maria Chico Granulitic Complex. Thus, protolith ages were assumed to be older than $2.55 \mathrm{Ga}$. However, the ages between $2.55 \mathrm{Ga}$ and the age of metamorphism may correspond to the age of the protoliths or partially reset ages. This was tested in this investigation. The interpretation by Hartmann et al. (1999) of protolith ages older than $2.5 \mathrm{Ga}$ is compatible with the idea by Friend and Kinny (1995), who defined the metamorphic age of the Lewisian Complex as the youngest Archean zircon U-Pb ion microprobe age obtained. These authors interpreted the spread of Archean ages as being the result of partial resetting of the magmatic zircon during a granulite facies growth event. The age of metamorphism for the Santa Maria Chico Granulitic Complex is suggested by the concentration of younger ages (2.1-2.0 Ga, Hartmann et al. 1999). Thus, the more complex isotopic relationships yielding older ages (this work) are associated to protoliths ages. The present investigation suggests that in this specific case the dating of protolith age of the granulite-facies rock can be done on associated amphibolite facies zircon crystals that underwent a high-grade metamorphic event at the same time as the granulites.

The amphibolite facies granodiorite gneiss crystallized from a magma at $2366 \pm 8 \mathrm{Ma}$ and some protoliths of granulite facies rocks in the complex were also probably formed at this time. The very intense metamorphic alteration of the granulite facies zircon caused the blurring of U-Pb ages in the entire crystal. Age interpretation of the granulite protolith is therefore liable to error, because the oldest zircon age from a sample is not necessarily the minimum age of the magmatic crystallization of the protolith (Friend and Kinny 1995, Hartmann et al. 1999). The present investigation suggests that the magmatic age of the granulite may be hidden in the array of partly reset, nearly concordant isotopic zircon compositions, and that the oldest ages may correspond to those of xenocrysts. A granulitic complex may be constituted by rocks formed in different, sequential geological events, and these may be dated by systematic investigation of associated coeval 

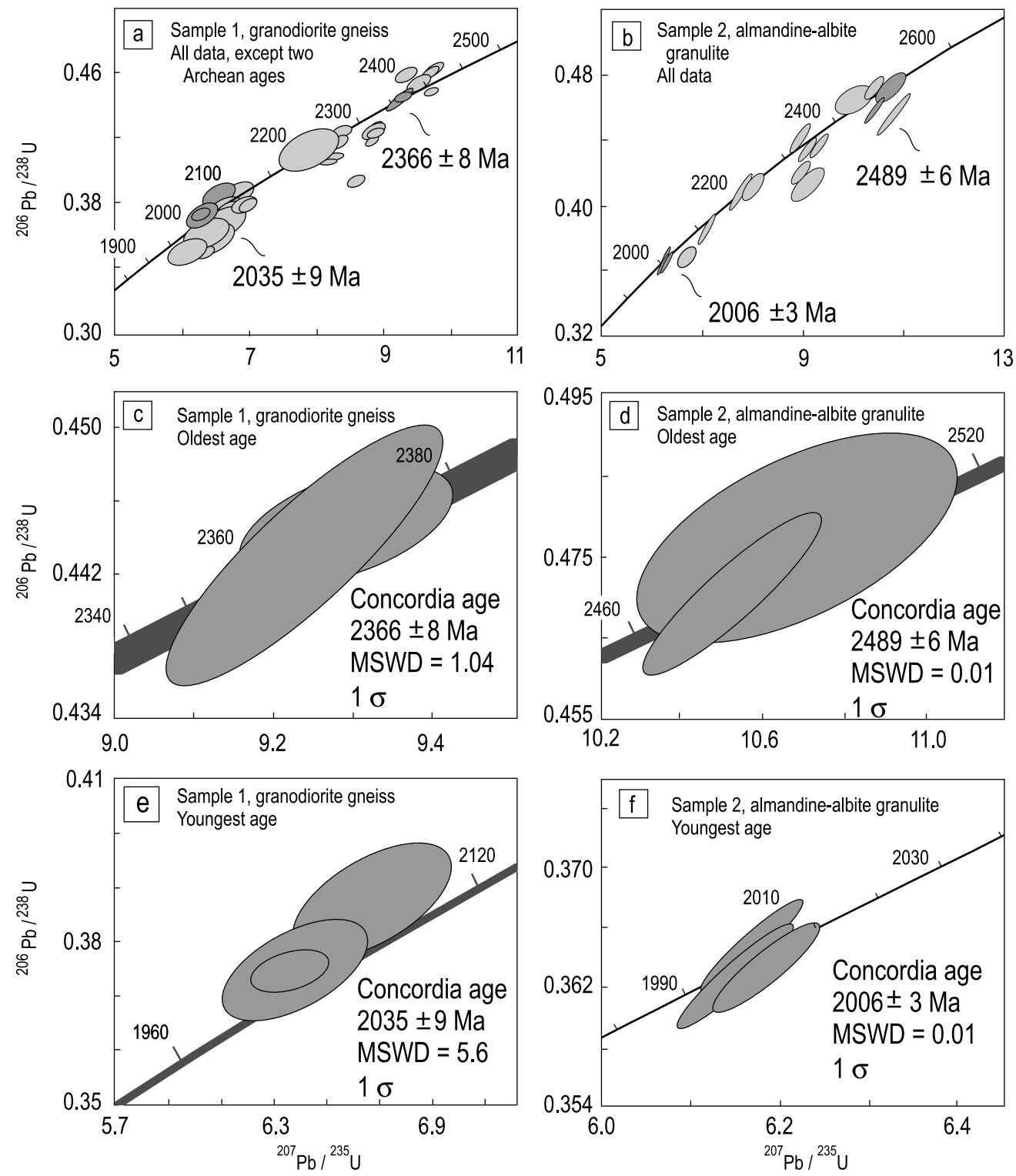

Fig. 3 - Concordia diagrams of dated samples 1, granodiorite gneiss (a, c and e) and sample 2, felsic almandine-albite granulite (b, $d$ and $f$ ). Oldest and youngest concordia ages of each sample shown.

amphibolite facies rocks whenever geological conditions are similar to those presently studied.

Amphibolite facies rocks may preserve the protolith ages of the associated granulite facies rocks, as in the present case, and this is relevant to severely deformed units such as the Santa Maria Chico Granulitic Complex $\left(800^{\circ} \mathrm{C}, 9-10 \mathrm{kbar}\right)$ in the southern Brazilian Shield. In other terrains, the geological relationship of the two rock types may be different from this study.
The heterogeneous evolution of the Santa Maria Chico Granulitic Complex is also reflected in the model Nd $\mathrm{T}_{\mathrm{DM}}$ ages (Table I), because they vary from ca. $2600 \mathrm{Ma}$ to ca. $2300 \mathrm{Ma}$. This indicates an orogenic evolution from the Late Archean to the Paleoproterozoic, now better delimited by the U-Pb dating of zircon crystals from one orthogneiss and one paragneiss presented here.

A granulite-facies metasedimentary rock (sample 2, 
almandine-albite granulite) from the Santa Maria Chico Granulitic Complex is dated for the first time in this investigation. The maximum age of sedimentation may correspond to the youngest detrital component (ca. $2200 \mathrm{Ma})$ or to the magmatic age of the granodiorite gneiss (2366 Ma). This is a significant contribution to the understanding of the Paleoproterozoic evolution of the southern Brazilian Shield.

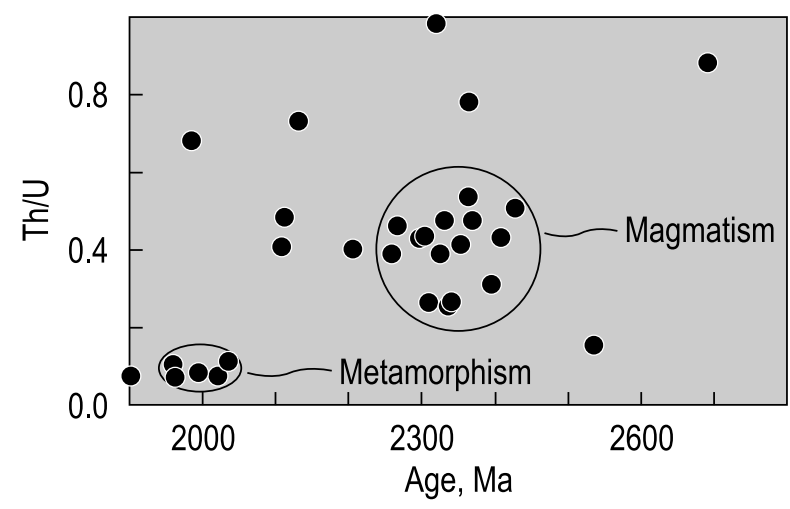

Fig. 4-Age versus Th/U ratio of zircon, sample 1, granodiorite gneiss. $\mathrm{Th} / \mathrm{U}$ ratios near 0.4 are considered magmatic and ratios $<0.1$ metamorphic.

The significance of ages in the $2200 \mathrm{Ma}$ range from both samples is not clear yet, because the available data do not allow the discrimination between the following explanations: (1) partially reset zircons or (2) intermediate metamorphic pulse. Additional investigations are required to understand the geological meaning of these ages.

Other amphibolite-facies granitic and metabasaltic rocks occur in the shield within $100 \mathrm{~km}$ of the studied rocks. These include the Encantadas Complex, which is covered by the deformed sedimentary sequence of the Porongos Complex. Provenance studies based on U$\mathrm{Pb}$ zircon SHRIMP ages from quartzites indicate an age spectrum closely comparable to the studied rocks, including ages near $2.35 \mathrm{Ga}$ (Hartmann et al. 2004). The Santa Maria Chico Granulitic Complex may be the deepcrustal equivalent of the mid-crustal Encantadas Complex gneisses. Also, Tickyji et al. (2004) recorded a Th*$\mathrm{Pb}$ electron microprobe age near $2.35 \mathrm{Ga}$ on monazite from a felsic garnet gneiss from the granulitic complex studied here. A geological event was dated in zircon by U-Pb TIMS at $2350 \pm 30 \mathrm{Ma}$ in the Santa Catarina
Granulitic Complex (Basei et al. 2000), situated $700 \mathrm{~km}$ to the north of the studied granulites. This makes this investigation more significant, because of the regional extension of the $2.36 \mathrm{Ga}$ magmatic rocks in the southern Brazilian Shield, which resided within Mesoproterozoic Supercontinent Columbia and Neoproterozoic Supercontinent Gondwana during the late Paleoproterozoic to early Neoproterozoic.

\section{ACKNOWLEDGMENTS}

This investigation was supported by Programa de Apoio a Núcleos de Excelência - Fundação de Apoio à Pesquisa do Estado do Rio Grande do Sul/ Conselho Nacional de Desenvolvimento Científico e Tecnológico (PRONEXFAPRGS/CNPq) on "Strategic Minerals from southern Brazil", Coordenação de Aperfeiçoamento de Pessoal de Nível Superior - BMBF/Federal Ministry of Education and Research - (CAPES-BMBF) cooperation project (BRA 00/44), and by the Chinese Adacemy of Geological Sciences. The SHRIMP II used for this investigation is housed at the Chinese Academy of Geological Sciences, Beijing, China.

\section{RESUMO}

Um granodiorito de fácies anfibolito e um almandina-albita granulito do Complexo Granulítico Santa Maria Chico, porção sul do Escudo Brasileiro, foram datados pelo método U-Pb em zircão por Beijing SHRIMP II (sensitive high resolution ion microprobe). Esta investigação inclui a determinação das idades de protólitos que estão ocultas no conjunto de dados parcialmente re-equilibrados. As idades metamórficas obtidas no gnaisse granodiorítico e no granulito são $2035 \pm 9 \mathrm{Ma}$ e $2006 \pm 3 \mathrm{Ma}$, respectivamente. Esses dados estão dentro da variação das idades metamórficas determinadas em estudos anteriores (2022 $\pm 18 \mathrm{Ma}$ e $2031 \pm 40 \mathrm{Ma})$. No entanto, as idades do protólito do granodiorito $(2366 \pm 8 \mathrm{Ma})$ e do granulito $(2489 \pm 6 \mathrm{Ma})$ estão fora da variação de idades (> 2510$2555 \mathrm{Ma}$ ) reconhecidas anteriormente. A idade magmática do protólito do granodiorito corresponde a uma idade pouco conhecida anteriormente no escudo. Estudos adicionais podem demonstrar que cristais de zircão de fácies anfibolito são úteis como janelas para o entendimento de eventos geológicos em granulitos associados, pois as idades de zircão nos granulitos estudados encontram-se obscurecidas por recristalização. 
Palavras-chave: idade de protólito, Paleoproterozóico, geocronologia de zircão, Complexo Granulítico Santa Maria Chico, SHRIMP.

\section{REFERENCES}

Basei Mas, Siga O, Masquelin H, Harara OM, Reis Neto JM And Preciozzi Porta F. 2000. The Dom Feliciano Belt and the Rio de la Plata Craton: tectonic evolution and correlation with similar provinces of southwestern Africa. In: Cordani UG, Milani EJ, ThomaZ FILHO A AND CAMPOS DA (Eds), Tectonic evolution of South America. 31st International Geological Congress, Rio de Janeiro, p. 311-334.

FRIEND CRL AND KINNY PD. 1995. New evidence for protolith ages of Lewisian granulites, northwest Scotland. Geology 23: 1027-1030.

GASTAL MCP AND LAFON J-M. 2001. Novas idades ${ }^{207} \mathrm{~Pb} /$ ${ }^{206} \mathrm{~Pb}$ e geoquímica isotópica $\mathrm{Nd}-\mathrm{Sr}$ para granitóides shoshoníticos e alcalinos das regiões de Lavras do Sul e Taquarembó, RS: VIII Congresso Brasileiro de Geoquímica, Curitiba, CD-ROM, 7 p.

HARTMAnN LA. 1998. Deepest exposed crust of Brazil Geochemistry of Paleoproterozoic depleted Santa Maria Chico granulites. Gondwana Res 1: 331-341.

HaRtmann LA, Leite JAD, MCNAUghton NJ AND SANTOS JOS. 1999. Deepest exposed crust of Brazil SHRIMP establishes three events. Geology 27: 947-950.

Hartmann LA, Leite JAD, Silva LC, Remus MVD, McNaughton NJ, Groves Di, Fletcher IR, SANTOS JOS AND VASCONCELlos MAZ. 2000a. Advances in SHRIMP geochronology and their impact on understanding the tectonic and metallogenic evolution of southern Brazil. Australian J Earth Sci 47: 829-844.

Hartmann LA, Santos JOS, MCNAUghton NJ, VASCONCELlos MAZ AND SILVA LC. 2000b. SHRIMP dates recurrent granulite facies metamorphism in the Santa Catarina granulites, southern Brazil. An Acad Bras Cienc 72: 559-572.

Hartmann LA, Philipp RP, LiU D, WAN Y, WANG Y, SANTOS JOS AND VASCONCELlos MAZ. 2004. Paleoproterozoic provenance of detrital zircon, Porongos Complex quartzites, southern Brazilian Shield. Int Geol Rev 46: $127-157$.
Massonne H-J, Tikovsky T And HaRtmann LA. 2001. Petrology of the 2.0 Ga old Santa Maria Chico granulites in southern Rio Grande do Sul, Brazil, and implications for crustal thickening in Paleoproterozoic times. XI Congr. Latinoamericano de Geologia, Montevideo 2001, Report, 4 p. on CD.

Möller A, O'Brien PJ, Kennedy A And Kröner A. 2003. The use and abuse of Th-U ratios in the interpretation of zircon. Geophys Res Abstracts 5: 12113.

SANTOS JOS, HARTMANN LA, BOSSI J, CAMPAL N, SCHIpilov A, Piñeyro D ANd McNaughton NJ. 2003. Duration of the Trans-Amazonian Cycle and its correlation within South America based on U-Pb SHRIMP geochronology of the La Plata Craton, Uruguay. Int Geol Rev 45: $27-48$.

SILVA LC. 2006. Geocronologia aplicada ao mapeamento regional, com ênfase na técnica U-Pb SHRIMP e ilustrada com estudos de casos brasileiros. Brasília, Publicações Especiais do Serviço Geológico do Brasil 1, 132 p. Available on Internet at www.cprm.gov.br.

Smith JB, Barley ME, Groves Di, Krapez B, Bickle MJ AND ChAPMANN HJ. 1998. The Scholl shear zone, west Pilbara: evidence for a terrane boundary from intergrated tectonic analysis, SHRIMP U-Pb age-dating and granitoid geochemistry. Precambrian Research 88: 143171.

SOLIANI JR E. 1986. Os dados geocronológicos do Escudo Sul-Rio-Grandense e suas implicações de ordem geotectônica. Doctoral Thesis, Instituto de Geociências, Universidade de São Paulo, 388 p.

Tickyji H, Hartmann LA, Vasconcellos MAZ, PhiLIPP RP AND REMUS MVD. 2004. Electron-microprobe dating of monazite substantiates ages of major geological events in the southern Brazilian shield. J South Am Earth Sci 16: 699-713.

VAVRa G, Schmid R AND Gebauer D. 1999. Internal morphology, habit and $\mathrm{U}-\mathrm{Th}-\mathrm{Pb}$ microanalysis of amphibolite-to-granulite facies zircons: geochronology of the Ivrea Zone (Southern Alps): Contrib Mineral Petrol 134: 380-404. 